\title{
Shape Coexistence in Proton Rich Se Isotopes
}

\author{
A. Mukherjee ${ }^{1}$, S. Bhattacharya ${ }^{1}$, T. Trivedi $^{1}$, R.P. Singh ${ }^{2}$, \\ S. Muralithar ${ }^{2}$, D. Negi ${ }^{3}$, R. Palit ${ }^{3}$, S. Nag $^{4}$, S. Rajbanshi ${ }^{5}$, \\ S. Kumar ${ }^{6}$, M. Kumar Raju ${ }^{7}$, D. Choudhury ${ }^{8}$, S. Sihotra ${ }^{9}$, \\ R. Kumar ${ }^{2}$, S.C. Pancholi ${ }^{2}$, A.K. Jain ${ }^{10}$
}

${ }^{1}$ Department of Pure \& Applied Physics, Guru Ghasidas Vishwavidyalaya, Koni, Bilaspur-495009, India

${ }^{2}$ Inter University Accelerator Centre, Aruna Asaf Ali Marg, New Delhi-110067, India

${ }^{3}$ Department of Nuclear and Atomic Physics,

Tata Institute of Fundamental Research, Mumbai-400005, India

${ }^{4}$ Department of Physics, IIT (BHU), Varanasi-221005, India

${ }^{5}$ Department of Physics, Presidency University, Kolkata-700073, India

${ }^{6}$ Department of Physics and Astrophysics, University of Delhi,

Delhi-110007, India

${ }^{7}$ Department of Physics, GITAM Institute of Science,

Vishakhapatnam-530045, India

${ }^{8}$ Department of Physics, Indian Institute of Technology, Ropar, Punjab-140001, India

${ }^{9}$ Department of Physics, Punjab University, Chandigarh-160014, India

${ }^{10}$ Amity Institute of Nuclear Science \& Technology, Amity University UP, Noida-201313, India

Received 15 October 2021

doi: https://doi.org/10.55318/bgjp.2022.49.1.108

\begin{abstract}
The structural features of the ${ }^{72}$ Se nucleus, obtained from our recent work [1], have been compared with the neighboring eveneven proton rich $\mathrm{Se}$ isotopes to get a better understanding about the presence of shape coexistence at low spin states. The corresponding alignment behaviour of different Se isotopes has been used to understand the shapes of the coexisting states at low excitation energy. The experimental results of Se isotopes have been interpreted in the framework of total Routhian surface (TRS) calculations.
\end{abstract}

KEY WORDS: Shape coexistence, TRS calculations.

\section{Introduction}

The quantal composition of atomic nuclei is susceptible to various symmetrybreaking phenomena influencing their structural behaviour. The nuclei lying in 


\section{Shape Coexistence in Proton Rich Se Isotopes}

the vicinity of transitional $A \approx 70$ mass region are of particular interest, which showcases a variety of phenomena viz. shape coexistence, octupole correlations, shape evolution, etc., due to the presence of competing shell gaps and high-j unique parity orbital. The nature of shape coexistence is well studied in the even-even nuclei having $N(Z) \approx 34$, 36. In ${ }^{74,76} \mathrm{Kr}$ nuclei, prolate-oblate shape coexistence has been confirmed using the spectroscopic quadrupole moment extracted from the precise lifetime measurements of the excited states [2]. The inversion of ground-state shape to oblate structure in ${ }^{72} \mathrm{Kr}$ was established from the relatively low values of $\mathrm{B}\left(E 2 ; 0_{1}^{+} \rightarrow 2_{1}^{+}\right)$measured using Coulomb excitation experiment [3].

In proton rich Se isotopes, the topic of coexisting shapes at low-spin states is quite sensitive. In $N=Z{ }^{68} \mathrm{Se}$ isotope, the oblate shaped ground-state band coexists with the prolate shaped excited band [4]. While, in $N=Z+2$

${ }^{70} \mathrm{Se}$ isotope, Hurst et al., predicted the prolate shape of the ground-state using the transition matrix element measured from the Coulomb excitation experiment [5]. The precise lifetime measurement and the corresponding transition strength $\mathrm{B}(E 2)$ contradicted the former prediction [6]. The relatively low values of $\mathrm{B}\left(E 2 ; 4_{1}^{+} \rightarrow 2_{1}^{+}\right)$and $\mathrm{B}\left(E 2 ; 2_{1}^{+} \rightarrow 0_{1}^{+}\right)$predicts that the ground-state band remains oblate up to $4^{+}$state and then shift towards prolate deformation. In ${ }^{72} \mathrm{Se}$ isotope, the first observation of shape coexistence was established by Hamilton et al. [7]. It was suggested that the ground-state band has a sphericalvibrator like structure, whereas the excited band built on $0_{2}^{+}$isomeric state has a deformed character. Further, $\mathrm{B}(E 2)$ values and spectroscopic quadrupole moment obtained from the lifetime measurement of the ground-state band show the quick evolution of oblate shaped ground-state towards prolate deformation after $2^{+}$state [6]. The theoretical calculations based on the adiabatic self-consistent collective coordinate (ASCC) method also support the experimental observations in ${ }^{68,70,72} \mathrm{Se}$ isotopes [8,9]. Further, in the ${ }^{74} \mathrm{Se}$ isotope, the coexistence of near-spherical vibrational states with prolate deformed states at low-lying structures has been predicted from the $\beta$-decay study of the ${ }^{74} \mathrm{Br}$ nucleus [10].

In the present study, a comparative study of ${ }^{68,70,72,74} \mathrm{Se}$ has been presented in the context of shape coexistence. The experimental observations are interpreted in terms of total Routhian surface (TRS).

\section{Experimental Data}

The excited states of ${ }^{72} \mathrm{Se}$ nucleus were recently populated using ${ }^{50} \mathrm{Cr}\left({ }^{28} \mathrm{Si}\right.$, $\alpha 2 p)^{72}$ Se reaction [1]. A detailed discussion of the experiment is mentioned in Refs. $[1,11,12]$. The present study is based on the comparison of structural features of different Se isotopes for which the experimental data have been taken from our work [1] and previous Refs. [4,13-16]. 


\section{A. Mukherjee et al.}

\section{Shape Coexistence}

The shape driving behaviour of $g_{9 / 2}$ orbital in $Z=34$ Se isotopes highly influences the coexisting shapes at low-lying states. Fischer et al. [4] confirmed the oblate deformation of the ground-state band (gsb) in ${ }^{68} \mathrm{Se}$ from the variation of kinematic moment of inertia. Whereas, the $\mathrm{B}(E 2)$ transition strength, measured from the lifetime of ground-state band in ${ }^{70} \mathrm{Se}$, shows a dominant oblate nature up to $4^{+}$state, which then evolves towards prolate deformation [6]. While in ${ }^{72} \mathrm{Se}$, the increasing values of $\mathrm{B}(E 2)$ with that of spin shows a quick evolution of the ground-state band from oblate to prolate structure [6]. One of the important signatures of shape coexistence is the presence of excited $0^{+}$ state, coexisting with the ground state, having different deformation. In ${ }^{68} \mathrm{Se}$ isotope although the $0_{2}^{+}$state has been predicted in Hartree-Fock-Bogolyubovbased configuration-mixing calculations [6], it has not yet been experimentally observed. The $0_{2}^{+}$state, observed at $937.2 \mathrm{keV}$ in ${ }^{72} \mathrm{Se}$ nucleus [17] is suggested to be the band-head of prolate deformed rotational band coexisting with the oblate-like ground state. In our recent studies on ${ }^{72} \mathrm{Se}$ [1], this $0_{2}^{+}$band has been extended up to $\left(10^{+}\right)$state along with the observation of several interconnecting $E 2(1431.4,1744.5$, and $1939.6 \mathrm{keV})$ and $E O(454.5,656.6,981.6 \mathrm{keV})$ transitions in between this band and the yrast band. The presence of such interconnecting transitions indicates the mixing between these two bands. While much information is not available for $0_{2}^{+}$state, observed at $2011.2 \mathrm{keV}$ in ${ }^{70} \mathrm{Se}$ nucleus [14]. The ${ }^{74} \mathrm{Se}$ isotope, having $0_{2}^{+}$state at $853.9 \mathrm{keV}$, is suggested to have near-spherical vibrational levels mixed with prolate deformed states at low excitation energies [10].

Further, Figure 1 shows the structural behaviour of ${ }^{72} \mathrm{Se}$ nucleus, which is compared with neighbouring even-even ${ }^{68,70,74} \mathrm{Se}$ isotopes in terms of the variation of their aligned angular momentum $\left(I_{x}\right)$ as a function of rotational frequency (in $\mathrm{MeV}$ ). The level energies for ${ }^{72} \mathrm{Se}$ isotope are taken from our present work as mentioned in Table 1 whereas for ${ }^{68,70,74}$ Se nuclei those have been taken from Refs. $[4,13,16]$ respectively. The low values of $I_{x}$ varying smoothly with spin in ${ }^{68} \mathrm{Se}$ exhibit its rotating oblate shape, which was also concluded by Fischer

Table 1. Spin and corresponding level energies of yrast band and excited $0^{+}$band in ${ }^{72} \mathrm{Se}$ obtained from our work [1].

\begin{tabular}{ccc}
\hline \hline Spin & $\begin{array}{c}\text { Level energies of yrast band } \\
\text { (in keV) }\end{array}$ & $\begin{array}{c}\text { Level energies of excited } \mathbf{0}^{+} \text {band } \\
\text { (in keV) }\end{array}$ \\
\hline $0^{+}$ & 0.0 & 937.0 \\
$2^{+}$ & 862.0 & 1316.4 \\
$4^{+}$ & 1636.7 & 2293.1 \\
$6^{+}$ & 2466.7 & 3380.5 \\
$8^{+}$ & 3424.8 & 4406.5 \\
$10^{+}$ & 4504.1 & 5473.5 \\
\hline \hline
\end{tabular}




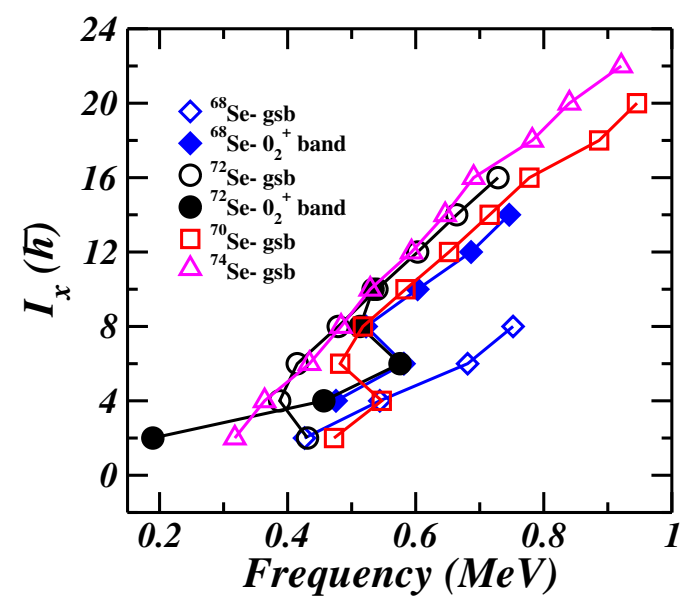

Figure 1. (Color online) Variation of aligned angular momentum $\left(I_{x}\right)$ as the function of frequency (in MeV) for the gsb of ${ }^{68,70,72,74} \mathrm{Se}$ nuclei.

et al. in Ref. [4]. A similarity has also been observed between the structure of the excited band in ${ }^{68} \mathrm{Se}$ and $0_{2}^{+}$band in ${ }^{72} \mathrm{Se}$. A back-bending for both the bands is observed at around $0.6 \mathrm{MeV}$, after which the excited band of ${ }^{68} \mathrm{Se}$ shows prolate-like structure [4]. The low-lying states of gsb in the ${ }^{70} \mathrm{Se}$ [13] isotope show significant perturbation while it behaves as a rotational band with constant deformation after $I \geq 6$ state. Similarly, the states lying below $4^{+}$in ${ }^{72} \mathrm{Se}$ [1] show a spherical vibrator-like structure, whereas it moves towards prolate deformation with increasing frequency. On the other hand, in ${ }^{74}$ Se nucleus [16], the aligned angular momentum of gsb exhibits a smooth behaviour indicating its prolate deformed nature. The experimental results of ${ }^{72} \mathrm{Se}$ are also consistent with various model based theoretical calculation $[6,8,9]$. Thus, from the above comparative discussion, it has been observed that ${ }^{70,72} \mathrm{Se}$ nuclei are transitional isotopes lying in between oblate ${ }^{68} \mathrm{Se}$ and prolate ${ }^{74} \mathrm{Se}$ nucleus.

\section{$4 \quad$ TRS Calculations}

The structural features of ${ }^{68,70,72,74}$ Se nuclei have been discussed using the total Routhian surface(TRS) calculations. The detailed methodology has been described in Ref. $[1,11]$. The $\beta_{2}-\gamma$ mesh is constructed from the total Routhian surface (TRS) calculations for the ground-state bands of ${ }^{68,70,72,74} \mathrm{Se}$ isotopes. The structural variation in the shapes of different Se isotopes at ground state frequency can be vividly seen in Figure 2. In ${ }^{68} \mathrm{Se}$ isotope, two potential minima with $\beta_{2} \approx 0.29$ and 0.24 have been observed at $\gamma \approx-54^{\circ}$, and $53^{\circ}$, respectively. Whereas in ${ }^{70} \mathrm{Se}$ isotope, three potential minima having $\beta_{2} \approx 0.30,0.30$, and 0.28 have been observed at $\gamma \approx-58^{\circ},+2^{\circ}$, and $+54^{\circ}$, respectively. Similarly 
A. Mukherjee et al.
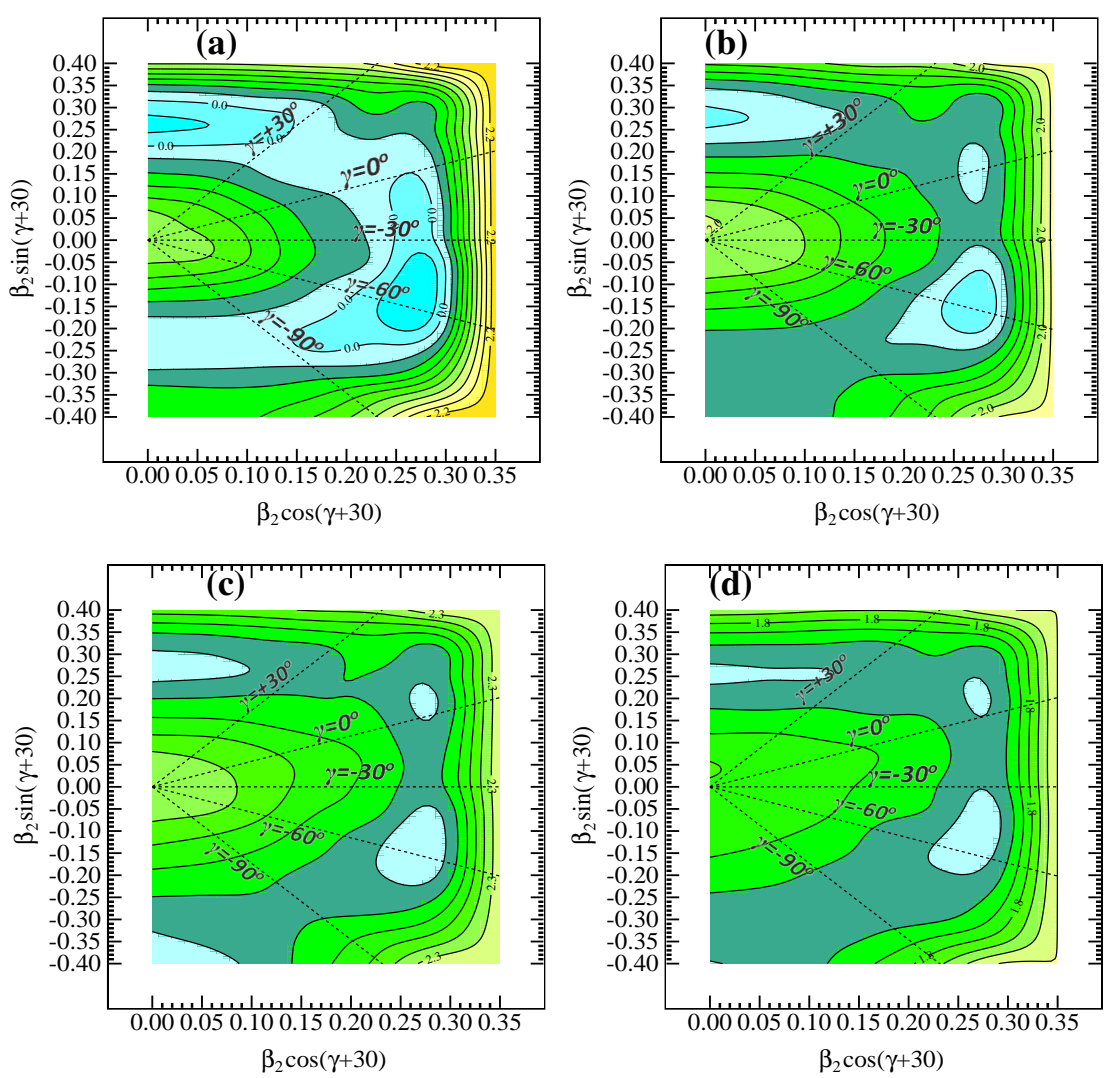

Figure 2. (Color online) Contour plot obtained from TRS calculations at frequency $0.05 \mathrm{MeV}$ for the positive parity yrast band in (a) ${ }^{68} \mathrm{Se}(\mathrm{b}){ }^{70} \mathrm{Se}$ (c) ${ }^{72} \mathrm{Se}$ and (d) ${ }^{74} \mathrm{Se}$, respectively.

in the ${ }^{72} \mathrm{Se}$ nucleus, three potential minima have been observed at $\gamma \approx-58^{\circ}$, $+2^{\circ}$, and $53^{\circ}$ having $\beta_{2} \approx 0.30,0.27$, and 0.32 respectively. These results show the coexistence of prolate and oblate shapes in ${ }^{70,72} \mathrm{Se}$ at the ground state configuration. Further, in the ${ }^{74} \mathrm{Se}$ nucleus, the non-collective minimum is observed at $\gamma \approx+53^{\circ}$ and the collective minimum is at $\gamma \approx-52^{\circ}$ whereas, the prolate minimum shifts toward $\gamma \approx+6^{\circ}$ with $\beta_{2} \approx 0.34$. Thus, the theoretically observed results are consistent with the experimental observation showing that the ${ }^{70,72} \mathrm{Se}$ are transitional nuclei lying in between dominant oblate ${ }^{68} \mathrm{Se}$ and dominant prolate ${ }^{74} \mathrm{Se}$ isotopes.

\section{Summary}

A comparative study has been made to establish the phenomenon of shape coexistence in different proton rich Se isotopes. The variation of aligned angular 112 


\section{Shape Coexistence in Proton Rich Se Isotopes}

momentum shows the transitional nature of ${ }^{70,72} \mathrm{Se}$ isotopes lying in between oblate ${ }^{68} \mathrm{Se}$ and prolate ${ }^{74} \mathrm{Se}$ nuclei. The TRS calculations have been used to describe the structural behaviour of ${ }^{68,70,72.74} \mathrm{Se}$ isotopes. Further, the consistency in the theoretically predicted results and experimental observation shows the variation in the nature of shape coexistence in different Se isotopes at groundstate frequency.

\section{Acknowledgement}

Authors acknowledge the INGA collaboration for establishing the INGA array and thank the Department of Science and Technology, Government of India, for providing funds for the INGA project (Grant No. IR/S2/PF-03/2003-I). Thanks to the staff of the target laboratory for facilitating the preparation of the target and the pelletron staff for smooth functioning of the accelerator at IUAC, New Delhi. A.M and S.B acknowledge the financial support from IUAC (UFR-63314) and UGC-DAE-CSR (UGC-DAE-CSR-KC/CRS/19/NP04/0915), respectively. A.K.J acknowledges the support from SERB (Govt. of India) in the form of grant CRG/2020/000770.

\section{References}

[1] A. Mukherjee et al., to be published.

[2] E. Clement et al. (2007) Phys. Rev. C 75054313.

[3] A. Gade et al. (2005) Phys. Rev. Lett. 95022502.

[4] S.M. Fischer, D.P. Balamuth, P.A. Hausladen, C.J. Lister, M.P. Carpenter, D. Seweryniak, J. Schwartz (2000) Phys. Rev. Lett. 844064.

[5] Hurst et al. (2007) Phys. Rev. Lett. 98072501.

[6] J. Ljungvall et al. (2008) Phys. Rev. Lett. 100102502.

[7] J.H. Hamilton et al. (1974) Phys. Rev. Lett. 32239.

[8] N. Hinohara, T. Nakatsukasa, M. Matsuo, K. Matsuyanagi (2009) Phys. Rev. C 80 014305.

[9] N. Hinohara, K. Sato, T. Nakatsukasa, M. Matsuo, K. Matsuyanagi (2010) Phys. Rev. C 82064313.

[10] E.A. McCutchan et al. (2013) Phys. Rev. C 87014307.

[11] S. Bhattacharya et al. (2019) Phys. Rev. C 100014315.

[12] S. Muralithar et al. (2010) Nucl. Instrum. Methods Phys. Res., Sect. A 622281.

[13] G. Rainovski et al. (2002) J. Phys. G: Nucl. Part. Phys. 282617.

[14] A. Ahmed et al. (1981) Phys. Rev. C 241486.

[15] P.D. Cottle et al. (1990) Phys. Rev. C 421254.

[16] J. Doring, G.D. Johns, M.A. Riley, S.L. Tabor, Y. Sun, J.A. Sheikh (1998) Phys. Rev. C 572912.

[17] E.A. McCutchan et al. (2011) Phys. Rev. C 83024310. 